\title{
Oscillations of body temperature at night
}

\author{
P J Brown, R A Dove, C S Tuffnell, R P K Ford
}

\begin{abstract}
There is increasing evidence that overheating is a contributing factor for some cot deaths. One hypothesis is that infant thermoregulation is closely related to respiratory control. To test this hypothesis it was necessary to determine the normal pattern of body temperature in the developing infant. A system has been designed and built to record continuously temperature signals from ambient, rectal, and various skin site sensors. Overnight studies were performed on 30 infants aged between 2 and 26 weeks in a hospital ward.

Various time and frequency domain analyses of the temperature data have been developed. Analysis of body temperature rhythms has confirmed patterns during sleep which mature with age. In addition a periodic oscillation of body temperature has been found with a cycle of approximately one hour. This oscillation may reflect sleep state and its further study may give an insight into control of infant thermoregulation and the integration of this control with that of breathing and the cardiovascular system.
\end{abstract}

\section{(Arch Dis Child 1992;67:1255-8)}

There is abundant evidence that there is a relation between cot death and temperature. Cot deaths occur more often in winter than in summer, ${ }^{1-4}$ more in higher latitudes in temperate zones, ${ }^{5}$ and overheating may be a contributing factor in many deaths. ${ }^{6-8}$ The physiological processes linking temperature changes to cot death have not been established.

Our hypothesis is that an increased body temperature, or thermal stress, is associated with increased respiratory instability. To test this hypothesis we first needed to determine the normal pattern of body temperature in the developing infant.

\section{Subjects and methods}

Our continuous temperature measurement studies were carried out in a hospital ward using a dedicated computer based polygraphic recording system (BabyLog). ${ }^{9}$ The ambient temperature of the hospital ward was $23-25^{\circ} \mathrm{C}$ throughout these studies, and all infants were lightly clothed in the equivalent of a cotton vest and a nappy. Infants were enrolled if they were considered afebrile by their doctor, did not have an infectious or inflammatory illness, and had no known encephalopathy. Recordings were completed on 30 such infants who had presented with minor choking episodes for which there was no obvious cause found on polysomnographic study. Their ages ranged from 2 to 26 weeks. Ethics committee approval and written parental informed consent were obtained for these studies.

The infants had continuous recordings of ambient and various body temperatures overnight for at least 12 hours. Rectal temperature was measured $5 \mathrm{~cm}$ from the anal margin and skin temperature from the forehead, axilla, wrist, abdomen, shin, and foot. We used temperature sensors which are based on a semiconductor rather than a thermistor. We have previously described the design, manufacture, and testing of these sensors, and the interface with our polygraphic recording system though a multiplexing isolating amplifier. ${ }^{10}$ The multiple temperature channels were sampled once a second and the signal data were stored on the computer system for later analysis. Sleep state, feeds, nappy changes, and other behaviour were recorded on a diary sheet and by continuous video monitoring.

Software has been written to produce a graphical output of the signal data and to allow measurement of any temperature recording at any sample point. Algorithms have also been developed for spectral analysis. ${ }^{11}$ These software algorithms process the temperature data in the Fourier domain to produce a screen output with peaks corresponding to dominant oscillations. The frequency, and hence period, of each of these peaks is determined on screen. The largest peak corresponds to the dominant frequency for a particular recording.

The temperature signals were than examined using the graphical software we have written for our system. Oscillations which varied in amplitude by more than $0 \cdot 1^{\circ} \mathrm{C}$ were measured for the length of period from peak to peak. These measurements of each identifiable period were then averaged (with $95 \%$ confidence intervals) for each recording.

Results

Thirty overnight studies have been examined in detail. Figure 1 presents an example of an overnight rectal temperature recording. Graphs of the continuous recordings showed trends on visual inspection that have been confirmed by further analysis.

\section{SLEEP-WAKE PATTERNS}

The variation in rectal temperature over a single night was $0.4-0.7^{\circ} \mathrm{C}$ in the younger infants (less than 8 weeks old) but was as much as $1.5^{\circ} \mathrm{C}$ in 


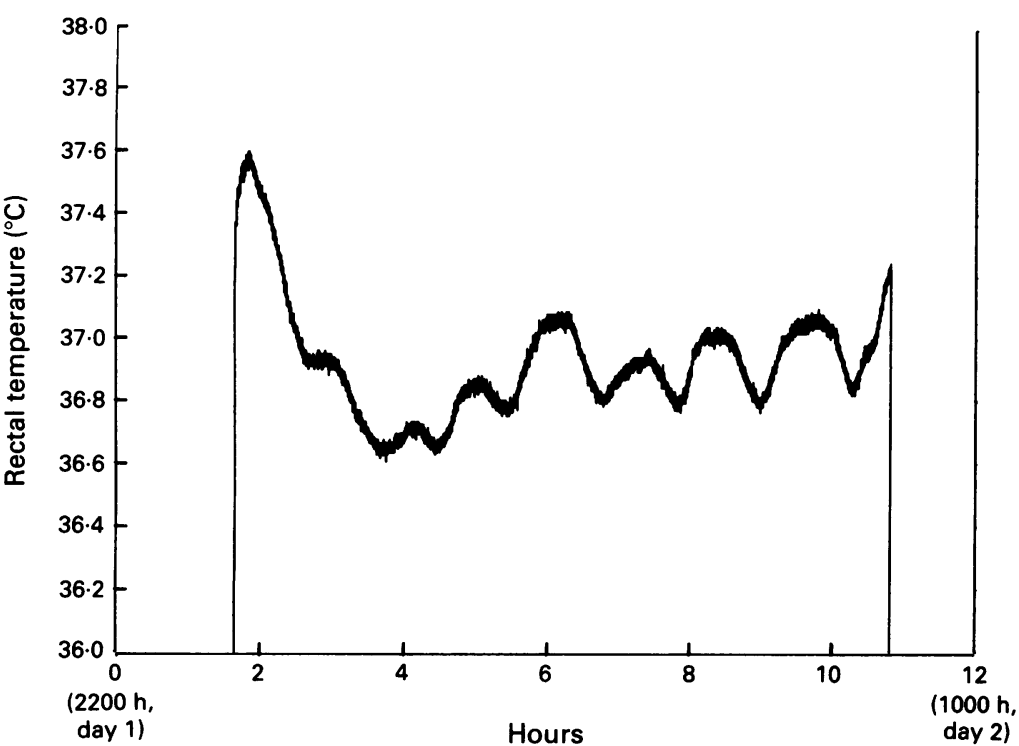

Figure 1 Continuous recording of rectal temperature in a 6 week old infant. Time in hours (from $2200 \mathrm{~h}$ ) on the $x$ axis and temperature $\left({ }^{\circ} \mathrm{C}\right.$ ) on the $y$ axis. Data were obtained once a second from bedtime until she was lifted from her cot in the morning. The graph shows the initial decrease in temperature, interrupted by a 'shoulder', then oscillations throughout the night with a cycle of approximately one hour.

those more than 12 weeks old. In an individual recording the temperature commonly varied from $37 \cdot 8$ to $36 \cdot 3^{\circ} \mathrm{C}$ through the night. When an infant went to sleep the rectal temperature decreased over about an hour to a nadir, then slowly increased until the infant woke for a feed or nappy change, when the temperature increased more quickly, and remained at this level until the infant went to sleep again. The decrease in temperature when sleeping after such a feed or nappy change was not as sudden as that seen on first going to sleep.

In infants under 4 weeks of age the axillary temperature reflected the rectal temperature fairly well, but in the older infants there was little similarity between axillary and rectal temperatures. The wrist, shin, and foot temperatures showed opposite trends to the rectal temperature, with a rapid increase of several degrees over about the first hour after falling asleep, and a rapid decrease when waking for a feed or nappy change. The forehead temperature followed neither the rectal nor the 'peripheral' pattern.

The extent and duration of this decrease in rectal temperature on first going to sleep was examined in relation to age for each infant (fig 2). The decrease was seen in the 26 infants older than 4 weeks but not in the four infants less than 4 weeks old. There was a tendency for the temperature to decrease more in older infants. There was little correlation of age with the duration of the decrease, which could take anything from 30 to 120 minutes (fig 2B; $r=0 \cdot 40$ ).

\section{RECTAL TEMPERATURE OSCILLATIONS}

In addition to the sleep-wake patterns described, we found a small amplitude oscillation of rectal temperature in 24 of the 30 continuous recordings. The amplitude of this oscillation was between 0.2 and $0.3^{\circ} \mathrm{C}$. The frequency of the oscillation was determined by two methods.
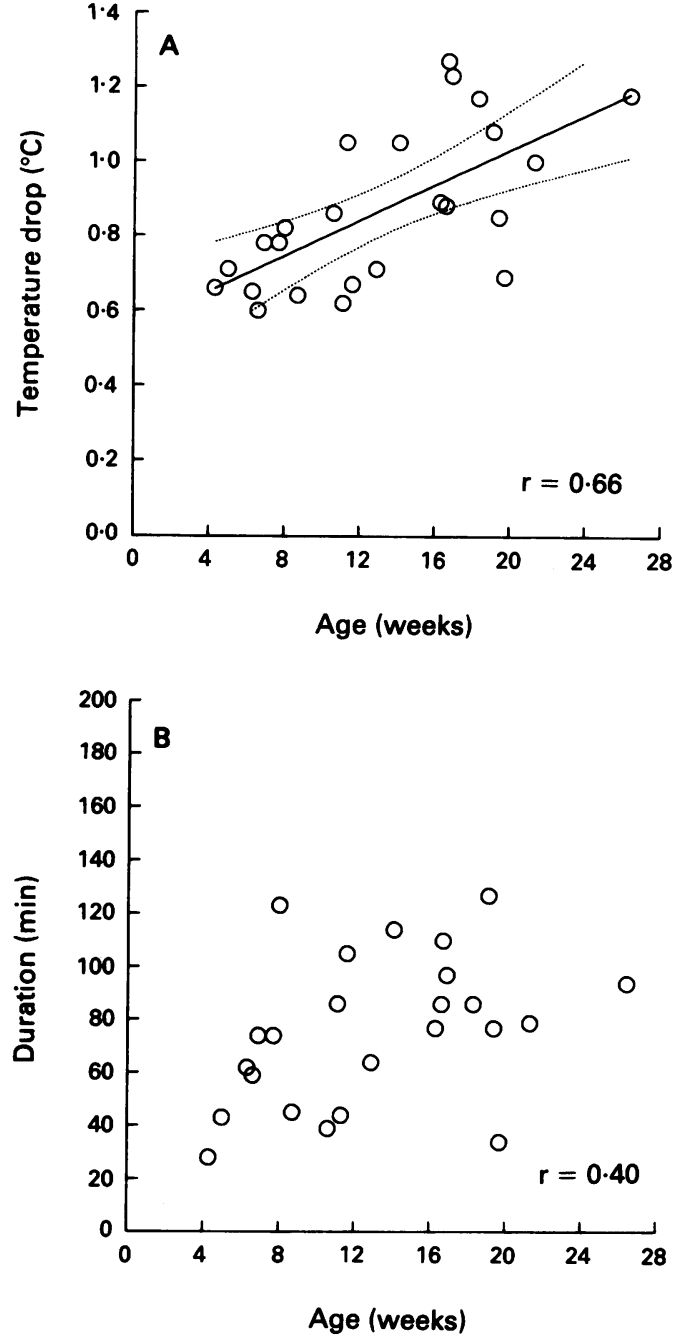

Figure 2 Characteristics of the decrease in rectal temperature after bedtime. $(A)$ Degree of the decrease related to age for each infant. A linear regression (with $95 \%$ confidence intervals) is fitted and has a coefficient of $0 \cdot 66$, showing a trend to a greater decrease in older infants.

(B) Duration of the decrease in temperature showing a poor correlation with age and a wide scatter lasting from 30 up to 120 minutes in different infants.

Spectral analysis was used to determine the dominant frequencies of any and all oscillations of the rectal temperature during the entire night of each recording. In six of 30 recordings no obvious spectral periods were identified. These six recordings were from infants less than 5 weeks old. In those infants more than 5 weeks old a sleep-wake cycle of three to four hours was identified and, in addition, spectral periods were found with a cycle length of approximately one hour. Figure $3 \mathrm{~A}$ presents these spectral periods in relation to age for each infant, showing the cluster around a one hour cycle length.

To define these oscillations more accurately, the raw signals from each rectal temperature recording were examined using computer software graphical methods. All distinct oscillations that could be identified (see fig 1) were measured. In six recordings distinct oscillations could not be found (the same six infants in whom spectral analysis did not identify obvious periods). In the 24 recordings from infants more than 5 weeks old the number of distinct 

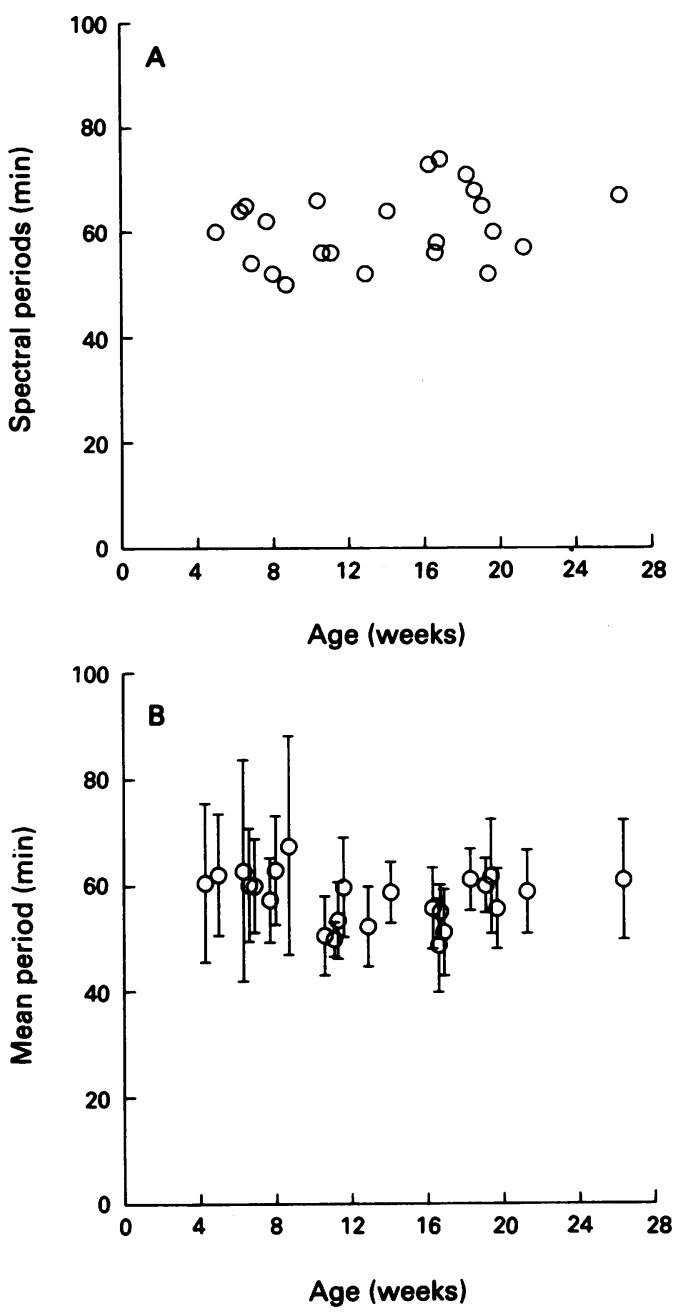

Figure 3 Two methods of determining the cycle length of the short term oscillations of rectal temperature related to the age of each infant. (A) Period of the frequencies detected by spectral analysis of each of the overnight recordings from spectral analysis of each of the overmight recordings from
bedtime until morming. $(B)$ Mean period and $95 \%$ confidence intervals of individual oscillations detected in each infant's recording. The two methods illustrate the approximately one hour cycle of these temperature oscillations.

oscillations varied between four and 12. The duration, or period, of each oscillation was measured and the mean period ( $95 \%$ confidence intervals) of the oscillations for each infant was calculated. Figure 3B presents these mean periods in relation to the age of each infant and shows the means clustered closely around one hour. The overall mean of the periods of the combined 24 studies was 58 (confidence interval $2 \cdot 1)$ minutes.

\section{Discussion}

Our hypothesis is that an infant's control of breathing is linked to temperature. To test this hypothesis we first needed to determine the normal pattern of body temperature in infants. As we are particularly interested in vulnerability to cot death, we focused on physiology during sleep in the developing infant during the first months of life.

Studies were undertaken in a hospital environment with a controlled ambient temperature and similar clothing insulation for all infants. These studies have shown certain patterns of body temperature and also proved that there is no reasonable alternative to measuring rectal temperature for continuous recording. A number of different skin sensors were used, including axilla, abdomen, forehead, wrist, shin, and foot, and none was found to correlate well with the rectal probe. ${ }^{10}$ Consideration was given to other methods of body temperature measurement such as tympanic membrane sensors or remote detection of microwaves. Techniques applicable in a hospital or laboratory environment are, however, unsuitable for long term use in a sleeping infant who is not under constant surveillance. We plan to extend our studies to the home environment and the requirements of reliability, durability, safety, and ease of use for parents guided our selection of equipment and techniques.

To determine patterns of body temperature accurately it is essential to measure continuously rather than take a few intermittent recordings. We chose to sample once a second, although once per minute would suffice. This sampling rate can produce much redundant data, but provides the facility to then use averaging algorithms or to search for frequencies of any period with spectral analysis without being limited by the basic sampling frequency. We report oscillations with a period of approximately one hour, but are able to identify other periodic changes (so called biological noise) with much higher frequencies.

This continuous sampling procedure and use of the entire data sets in analysis has uncovered short term oscillations of rectal temperature with a period of about 60 minutes. Other workers have also measured overnight rectal temperatures in infants and shown the initial decrease on going to sleep. ${ }^{12}{ }^{13}$ They did not observe the hourly oscillations seen in our studies, however, probably because of the method with which they produced their data sets. Although their temperatures were logged once a minute, for analysis purposes they used temperatures only every 15 or 30 minutes. The data set becomes much easier to handle, but with effective sampling at this frequency it would not be possible reliably to detect oscillations with a period of an hour or less.

We have confirmed several of the findings of these workers. The initial decrease in rectal temperature on going to sleep is a significant feature (fig 1 and 2). It appears in infants older than 4 weeks and its amplitude seems to increase in older infants, being as much as $1.3^{\circ} \mathrm{C}$ in those over 16 weeks. The duration of the decrease is not related to age and it is variable from less than 30 minutes to more than two hours. This variability in duration would be missed by sampling only every 15 or 30 minutes. The decrease itself is sometimes smooth to the nadir, but in some recordings is interrupted by a small 'shoulder' or rise, which appears to have the same amplitude and periodicity as the short term oscillations we have discovered.

Wailoo and coworkers took an arbitrary zero time for their analysis as the time of placing the infant in the cot, which ignores possible effects of any circadian rhythm due to the time of day but allows the determination of a pattern which 
seemed 'phase locked' to bedtime. ${ }^{12} 13$ Our studies have confirmed that the temperature decrease on sleeping seemed obligatory and independent of bedtime.

Two different methods of analysis of our study data indicate that there is an oscillation of rectal temperature with a period of approximately one hour (fig 3). The cause and significance of these short term oscillations is intriguing. They seem to become apparent after the first month of life, at the same age as the appearance of sleep-wake patterns described as 'mature', with a definite initial decrease in temperature 'phase locked' to bedtime. ${ }^{12} 13$

Sleep state changes with a cycle of approximately one hour have been described in a 2 month old infant with alterations in the variability of heart rate and respiratory rate coincident with electroencephalogram and electromyogram changes. ${ }^{14}$ Regularity in sleep alternation in a 3 month old infant has been shown with a cycle of about one hour. ${ }^{15}$ This age is a time of maximum concordance between physiological variables, higher than at any earlier or later age. Electroencephalogram parameters develop a rhythmicity with approximately a one hour cycle after the first week of life and coupling of this rhythmicity with other physiological variables (respiratory and cardiac) begins to emerge at 3 to 4 months of age. ${ }^{16}$ The hourly cycle of rectal temperature oscillations is therefore likely to be related to these sleep state changes, showing similar development with age. It may reflect phases of active sleep and indicate an increase in metabolic rate.

We plan to extend our studies to the home environment, ${ }^{11}$ to examine respiratory and cardiac signals to define sleep states, ${ }^{14}$ and any link between control of breathing and temperature. Analysis of these home recordings may allow us to determine the effects of thermal stress, both exogenous (ambient temperature, clothing, and bedding insulation) and endogenous (immunisations and infections), and to find any developmental changes in the individual infant over the first few months of life.

\section{CONCLUSION}

We have developed a computer based polygraphic recording system and modified it for future use in the home environment. Sampling at a high frequency has allowed analysis of continuous body temperature recordings. The rectal temperature rhythms of infants in the first six months of life show a previously unreported oscillation with a cycle of approxi- mately one hour. This becomes apparent at around the same age as the maturation of a recently described pattern of rapid decrease in temperature after bedtime.

With the addition of respiratory and cardiac signal recordings we plan to investigate these oscillations and other rhythms to determine their relation with behavioural or sleep state and the effects of changes in age, ambient temperature, clothing, and bedding, immunisations and illness. We may also be able to examine the integration of control of thermoregulation with that of breathing and the cardiovascular system, particularly during sleep at the most vulnerable age for cot death.

Dr Brown was supported by the Canterbury Cot Death Research Fellowship and Mr Tuffnell by the Aoraki Corporation. Grants were also received from Lotteries Medical Research. Mrs J Larkin gave invaluable assistance with the overnight temperature studies.

1 Golding J, Limerick S, Macfarlane A. Sudden infant death: patterns, puzzles and problems. Shepton Mallet: Open Books, 1985

2 Froggatt P, Lynas M, MacKenzie G. Epidemiology of sudden unexpected death in infants (cot death) in Northern Ireland. British foumal of Preventive and Social Medicine 1971:25:119-34.

3 Murphy MFG, Campbell MJ. Sudden infant death syndrome and environmental temperature: an analysis using vital and environmental temperature: an analysis using vital

4 Campbell MJ. Sudden infant death syndrome and environmental temperature: further evidence for a time-lagged mental temperature: further evidence for

5 Nelson EAS, Taylor BJ. Climatic and social associations with postneonatal mortality rates within New Zealand. $N Z$ Med F 1988;101:443-6.

6 Stanton AN. Overheating and cot death. Lancet 1984;ii: 1199-1202.

7 Nelson EAS, Taylor BJ, Weatherall IL. Sleeping position and infant bedding may predispose to hyperthermia and the sudden infant death syndrome. Lancet $1989 ;$;:199-200.

8 Fleming PJ, Gilbert R, Azaz Y, et al. Interaction between bedding and sleeping position in the sudden infant death syndrome: a population based case-control study. $B M \mathcal{J}$ 1990;301:85-9.

9 Dove R, Brown J, Fright R, Tuffnell C, Ford R. Computer polygraphic system for infants at risk for sudden infant polygraphic system for infants at risk for sudden infant death synd $188-91$.

10 Brown J, Dove R, Price B, Fong S, Ford R. Continuous multiple location body temperature measurement of infants. multiple location body temperature measurem

11 Ford RPK, Brown PJ, Dove RA, Tuffnell CS, Macey PM. HomeLog: long term recording of infant temperature, respiratory and cardiac signals in the home environment. $\mathcal{f}$ Paediatr Child Health (in press)

12 Wailoo MP, Petersen SA, Whittaker $\mathbf{H}$, Goodenough P. Sleeping body temperatures in 3-4 month old infants. Arch Dis Child 1989;64:596-9.

13 Lodemore M, Petersen SA, Wailoo MP. Development of night time temperature rhythms over the first six months of night time temperature rhythms o

14 Harper RM, Schechtman VL, Kluge KA. Machine classification of infant sleep state using cardiorespiratory measures.
Eléctroencephalogr Clin Neurophysiol 1987;67:379-87.

15 Hoppenbrouwers T. Sleep in infants. In: Guilleminault C, ed. Sleep and its disorders in children. Raven Press: New Yod. Sleep 1987

16 Harper RM, Leake B, Miyahara L, et al. Development of ultradian periodicity and coalescence at 1 cycle per hour in electroencephalographic activity. Exp Neurol 1981;73: 127-43. 